\title{
The Physics of Evaporation-Induced Assembly of Sol-Gel
}

\section{Materials}

\author{
Alan J. Hurd ${ }^{1}$ and Lev Steinberg ${ }^{2}$
}

RECEIVED

AUG $1 ? 2080$

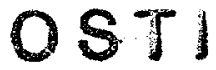

Abstract Remarkable materials ordered at the nanoscale emerge when a sol-gel solution becomes co-organized with a surfactant. At sufficiently high concentration, the surfactant forms crystalline or liquid-crystalline arrays of $\mathrm{mi}$ celles in the presence of the sol-gel, and as gelation proceeds the arrays become locked into the gel. Recent experiments demonstrate that the degree of order in the resulting mesoporous ceramic phase can be enhanced and controlled by continuous dip coating in which the solution, initially dilute, evolves through the critical micelle concentration by steady-state evaporation. The long-range order and microstructural orientation in these films suggest that the propagation of a critical-micelle-concentration transition front, with large physico-chemical gradients, promotes oriented self assembly of surfactant aggregates. This "steep-gradient" view is supported by results from un-

Received: now / Revised version: then

Alan J. Hurd and Lev Steinberg ${ }^{2}$

${ }^{1}$ Sandia National Laboratories

Albuquerque, New Mexico 87185-5000 USA

ajhurd Qsandia.gov,

${ }^{2}$ Department of Mathematics

University of Puerto Rico

Mayaguez, Puerto Rico 00681-5000 USA

s_lev@rumac.upr.clu.edu steady evaporation of aerosols of similar solutions, in which internally well-ordered but complex particles are formed.

1

\section{Introduction}

In evaporation-induced self-assembly[1] (EISA), sol-gel soJutions containing amphiphilic molecules become progressively concentrated through solvent evaporation thereby passing through structural and connectivity transitions that enable the rapid formation of nanoscopic materials. [14] As the solution becomes concentrated, the first transition that is important is the critical micelle concentration (CMC), in which the amphiphiles aggregate together into micellar or lamellar structures. Upon further evaporationinduced crowding, these aggregates interact to form mesophases whose structure is determined-assuming there is time for equilibrium-by the phase behavior of the solvent(s) and surfactant. Finally, there is gelation of the sol-gel species, effectively locking into place the mesophase structure as evinced by its presence after complete evaporation of solvent. These transitions are shown schematically in Fig 1; they take place over seconds or fractions of a second.

While much of the critical phenomenology of EISA has yet to be established, the influence of this rapid en- 


\section{DISCLAIMER}

This report was prepared as an account of work sponsored by an agency of the United States Government. Neither the United States Government nor any agency thereof, nor any of their employees, make any warranty, express or implied, or assumes any legal liability or responsibility for the accuracy, completeness, or usefulness of any information, apparatus, product, or process disclosed, or represents that its use would not infringe privately owned rights. Reference herein to any specific commercial product, process, or service by trade name, trademark, manufacturer, or otherwise does not necessarily constitute or imply its endorsement, recommendation, or favoring by the United States Government or any agency thereof. The views and opinions of authors expressed herein do not necessarily state or reflect those of the United States Government or any agency thereof. 


\section{DISCLAIMER}

\section{Portions of this document may be illegible in electronic image products. Images are produced from the best available original document.}




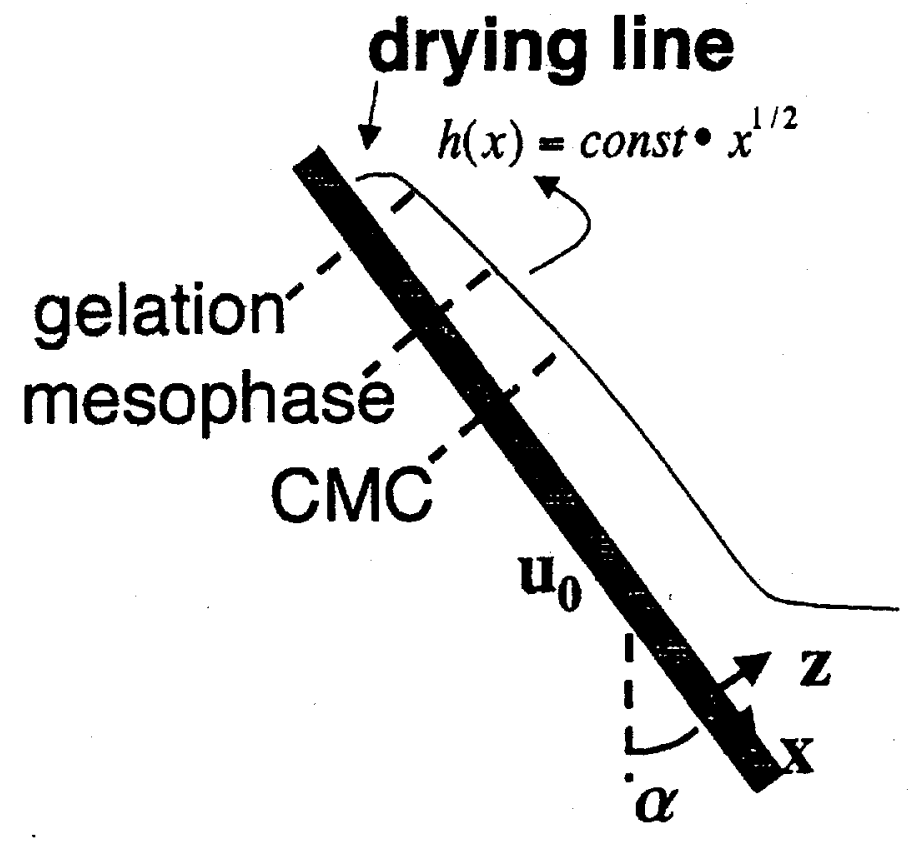

Fig. 1. The origin of aligned, ordered porosity in thin films by Evaporation-Induced Self-Assembly (EISA) is in sequential transitions within a drying liquid film in dip coating. Those transitions include the $\mathrm{CMC}$ in which surfactants aggregate into micelles, a transition from isotropic to a mesophase, anc gelation of the sol to lock the mesophase structure in place.

vironmental change appears to enhance structural order: In thin films made by dip coating, the boundaries (vapor and solid) of the liquid environment impose alignment of the surfactant structures, and the presence of steep gradients in concentration and, possibly, flow velocity may align the assembling material along a preferred direction. Quite possibly the passage of steep gradients through the liquid promotes self assembly and long-range order, informally called the "zipper hypothesis." The ordering may also be at work in aerosol EISA as observed in radially layered particles that have been made.[2] The zipper hypothesis is the essential point of this work and will be addressed from the standpoint of hydrodynamic shear and soiute concentration.
The experiment to be analyzed is performed by dip coating a substrate from a dilute silica sol-gel-surfactant solution made up with volatile solvents. By steadily withdrawing the flat, wettable substrate from the solution at velocity $u_{0}$, a steady state is reached at which the entrained liquid film recedes by evaporation at the same velocity as the withdrawal. A characteristic of the liquid film is its abrupt termination about $1 \mathrm{~cm}$ from the solution reservoir at a steady drying line, beyond which the deposited material forms a solid film. The entrained liquid has thickness of order micrometers but tapers to zero at the drying line in a characteristic way given empirically by

$h(x) \propto x^{1 / 2}$.

After deposition, the film typically consists of ordered mesophase structures of surfactant surrounded by a silica framework; a film may be rendered porous by heating to burn out the surfactant inolecules. Representative structures are shown in Fig 2 where the withdrawal is depicted at an angle $\alpha$ with respect to the vertical (for generality).

A successful theory of the liquid-film hydrodynamics would predict a drying line of order one centimeter from the reservoir. a thickness in the range of a few micrometers, and a profile matching Eq 1. A feature to be predicted is the magnitude and direction of shear in the liquid to see whether it is significant for mesophase alignment.

2.

\section{Physics of Dip Coating.}

A prerequisite to understanding the interplay of the critical micelle concentration (CMC) transition, mesophase as- 


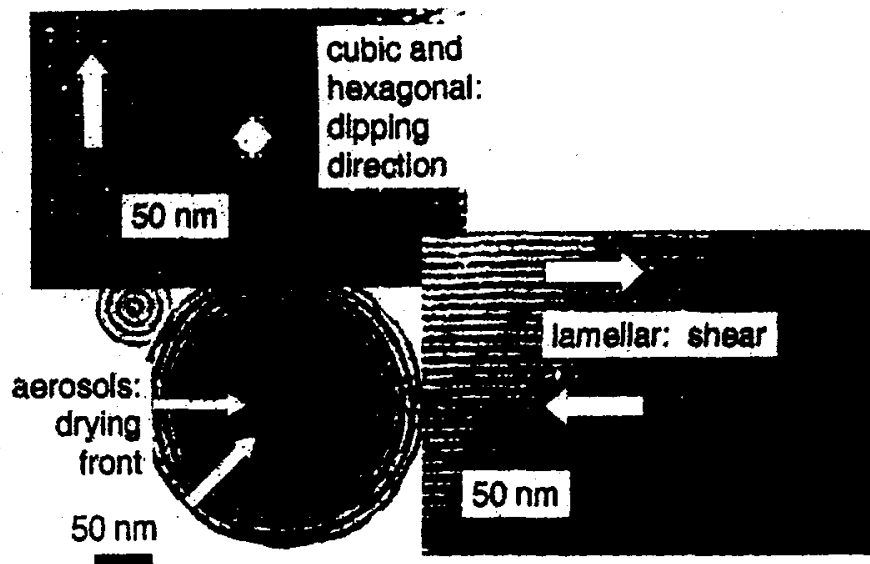

Fig. 2. Nanoscale order is produced in silica materials by EISA process. A cubic structure (top) is aligned with the dip costing withdrawal direction; a lamellar structure (lower right, crosssectional view) shows alignment to boundaries and shear if present; an aerosol particle (lower left) structure implies sequential shell formation.

sembly, and subsequent gelation is knowledge of the local environment of a fluid element. A quantitative description of the continuously evolving environment in a dip-coated film is sketched here from hydrodynamics and capillarity, paying due attention to the relative contributions of competing forces; a complete manuscript is in preparation.

The dominant feature of steady-state dip coating is the drying line, beyond which almost all volatile matter has evaporated leaving the deposited film. For a plane of liquid approaching the drying line, evaporation accelerates in a way that is characteristic of a planar source of vapor ending in an edge: Being a geometric effect, this evaporation behavior differs qualitatively for film, aerosol and fiber formation.

In the planar case, an ayproximate treatment shows that the evaporation rate diverges as $x^{-1 / 2}$ where $x$ is the distance from the drying line.[5] Briefly, the argument is that the vapor phase provides diffusive resistance to solvent evaporation, and the evaporation rate from a position far from the edge is small because a solvent molecule has only one preferred direction (perpendicular to the surface) to diffuse. On the other hand, a molecule at the edge of a liquid film has a range of possible departure angles, from the tangent to the substrate to the perpendicular direction. That additional freedom can be quantified by the steady-state diffusion equation with appropriate boundary conditions and lead to the $x^{1 / 2}$ behavior.

While thermal gradients may generally be neglected in films owing to the heat reservoir provided by the substrate, curvature-induced pressure $p(x, z)$ and concentration-induced osmotic pressure $\Pi(x, z)$ may be significant. These factors are governed by the Navier-Stokes equations, but in the very thin environment of the entrained film, it is legitimate to take the lubrication approximate form,

$-\frac{\partial p}{\partial x}+\eta \frac{\partial^{2} u}{\partial z^{2}}+\rho g \cos (\alpha)=0$,

$-\frac{\partial p}{\partial z}+\frac{\partial \Pi}{\partial z}-\rho g \sin (\alpha)=0$,

$u(x, z=0)=-u_{0}$

$\frac{\partial u}{\partial z}(x, z=h(x))=0$

$p(x, z=h(x))=-\gamma \kappa(x)$

$\kappa(x)=\frac{h^{\prime \prime}(x)}{\left[1+h^{\prime}(x)^{2}\right]^{3 / 2}}$.

Here $\kappa(x)$ is the curvature of the film of height $h(x)$. The only significant component of velocity $u(x, z)$ is in the $x$ direction; the $z$ component is negligible. The viscosity $\eta$ and surface tension $\gamma$ are taken to be constant in this review, although in practice they would be dependent on concentration of solvents and solutes. A quantity 
needed is the evaporation function $E(x)=E_{0} e(x)$ with $e(x) x^{1 / 2}$ representing the mass evaporated per unit time (per unit area of liquid-vapor interface), and $E_{0}$ is an essential dimensional quantity for the problem. Thus, the three basic quantities are $\eta, \gamma$, and $E_{0}$, and three characteristic lengths can be defined from them: a viscous length $d=\left(\eta u_{0} / \rho g\right)^{1 / 2}$ of order $10 \mu m$, a capillary length $D=(\gamma / \rho g)^{1 / 2}$ approximately $1 \mathrm{~mm}$, and a drying length $X_{0}=\left(\rho u_{0} / E_{0}\right) d$ that is a few $m m$.

The solution to these equations to lowest order in the parameter $\beta=D_{l} /(3 C a)^{1 / 3}$, where $D_{l}=E_{0} / \rho u_{0}$ is a dimensionless "drying" number and $C a$ is the capillary number $\eta u_{0} / \gamma$, is a cubic function in evaporative flux, determined by balancing the evaporative and hydrodynamic flux. Only the results of this program will be shown here.

3

\section{Results.}

In Fig. 3, the thickness $h(x)$ scaled by $d=\left(\eta u_{0} / \rho g\right)^{1 / 2}$ is plotted against the distance from the drying line scaled by $X_{0}$. The general features of dip coating a film are seen. including the empirical shape Eq. 1. From this solution, the velocity field and therefore the shear can be found; the latter is plotted in Fig. 4.

The magnitude of the shear rate is set by the value $u_{0} / d=500 s^{-1}$. It can be seen from Fig. 4 that the maximum shear occurs within $0.02 X_{0}$ from the drying line at the surface of the substrate and reaches a value of order $200 s^{-1}$. A portion of this shear is from gravitational backflow, which can be seen to be $50 \mathrm{~s}^{-1}$ near the center of the film, but the larger fraction is from capillary backflow re-

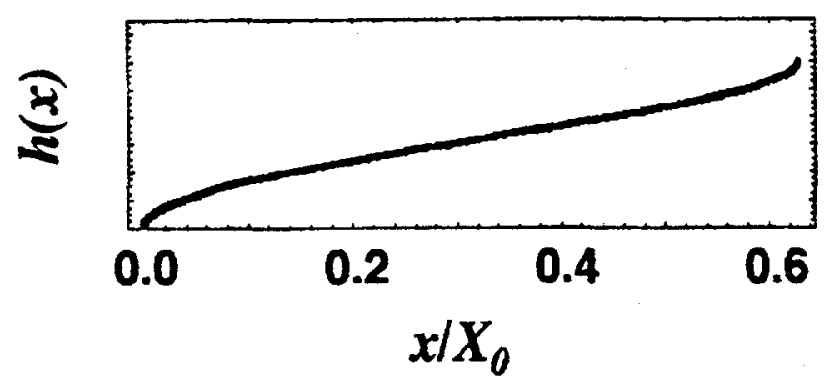

Fig. 3. Calculated film profile depicts parabolic shape near drying line and a gocd match to experimental thickness and drying line height.

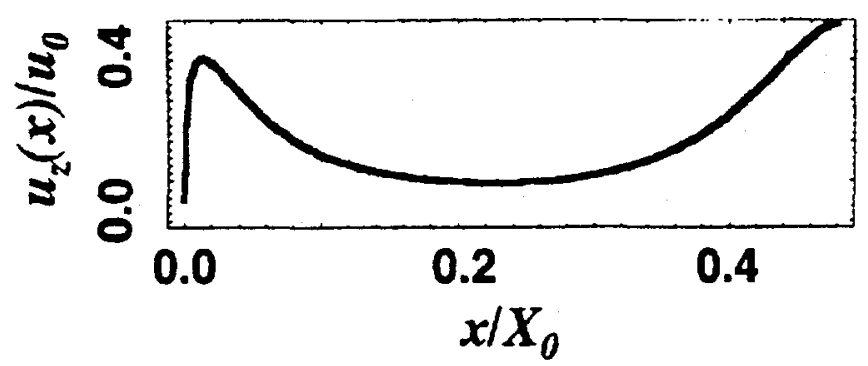

Fig. 4. Calculated shear profile in dip coating. The shear rate peaks near the drying line where film curvature, induced by evaporation dynamics, drives backflow. The shear is significant in alignment during assembly of structures.

sulting from the positive pressure bulge under the curved liquid film profile. (That curvature can be seen near the drying line in Fig. 3.) This shear is strong enough to lead to substantial flow alignment in mesophases, including lyotropic liquid crystals of the type at hand, while being capable of delaying gelation.

It is important to note that this analysis ignores concentration effects, such as changes in viscosity or surface tension. Both effects are known to be important in actual depositions, leading to measurable departures from the parabolic profile Eq. 1 for example; this theory provides a scaffold upon which to base an analysis of them. For insight into EISA, it is sufficient to consider only the 
concentration gradients implied by the evaporation rate and resulting film thickness profile. When the profile $h(x)$ follows Eq. 1, then the local concentration of involatile species varies as $h(x)^{-1} \sim x^{-1 / 2}$. (The assumption is made here that diffusion across the liquid film thickness homogenizes the concentration across streamlines, which is only approximately true in practice.) Thus the concentration gradient scales as $x^{-3 / 2}$, a relatively abrupt function. Adding to the shear, this singularity in concentration intensifies physicochemical stresses near the drying line.

4

\section{Conclusion.}

One has the developing picture of evaporation-induced self assembly as a highly cooperative transition with a number of interesting effects traceable to the evaporation singularity at the drying line. Hydrodynamic shear arises from capillary pressure near the drying line, which in turn de rives from the evaporation physics: liquid "burns off" at an accelerating rate as it approaches the drying line causing a parabolic blunting of the film profile. The resulting capillary pressure bulge drives backflow with a shear rate that is sufficient to cause two additional effects.

First, the self-assembling mesophase will align in a shear flow assuming it has a sufficiently anisotropic nature, such as lamellae or hexagonal packings of cylinders. It is suspected but unproven that this shear facilitates sequential assembly of surfactant structure and aligns the structure with respect to the dipping direction. Note that the critical micelle concentration is expected to have been passed through relatively early in the dipping sequence and with little fanfare. Second, the sol-gel phase is driven strongly toward gelation given the concentration gradients, but one expects the shear rate to prevent long-range gel clusters to form. In a sense, the sol-gel is "supercooled" and will abruptly "jam up" as soon as the shear rate falls below a certain threshold (probably about $20 \mathrm{~s}^{-1}$ ). It may be that the delayed gel transition causes the mesoporosity to be quenched quickly as the shear rate drops below the level at which gel clusters span the film thickness.

Acknowledgments. The authors thank Jeff Brinker, Yunfeng Lu and Frank van Swol for stimulating discussion of this research. This work was supported by Sandia National Laboratories, a Lockheed-Martin company under Department of Energy Contract DE-AC04-94AL85000, and by the Office of Basic Energy Sciences of the Department. of Energy.

\section{References}

1. Brinker CJ, Lu YF, Selinger A, Fan HY Advanced Materiais $11,(1999) 579-585$.

2. Lu YF, Fan HY, Stump A, Ward TL, Rieker T, Brinker CJ Nature 398, (1999) 223-226.

3. Lu YF, Cao GZ, Kale RP, Prabakar S, Lopez GP, Brinker CJ Chemistry Of Materials 11 (1999) 1223-1229.

4. Lu YF, Ganguli R, Drewien CA, Anderson MT, Brinker CJ, Gong W, Guo Y, Soyez H, Dunn B, Huang MH: Zink JI, Nature 389, (1997) 364-368.

5. Hurd AJ and Brinker CJ, Materials Research Socieiy Symposium Proceedings 180, (1990) 575-581. 